One great principle of the treatment of post-partum haemorrhage is that the left hand must never leave the fundus until all danger of bleeding has passed, and the uterus is contracting and retracting firmly and well.

Steps can now be taken to maintain contraction and retraction of the uterus. A hypodermic injection of ergometrine or pitocin is an excellent aid. If intra-uterine manipulations have been carried out, either manual removal or bi-manual compression, an intra-uterine douche of weak antiseptic at a temperature of $I I 8^{\circ} \mathrm{F}$. is valuable as a prophylactic against sepsis, as a stimulus to uterine contraction, and as an aid to the clotting of blood.

\title{
After-treatment.
}

If a large amount of blood has been lost, the obviously ideal treatment is to replace it immediately by a blood transfusion; but a blood transfusion can only be carried out when there is adequate assistance and facilities available. Less assistance is needed for a saline infusion, but usually in the circumstances of domiciliary practice this method is also not possible. However inadequate assistance and facilities are, it is always possible to insert fluid into the rectum and ten ounces of water from the pump in the garden, with half teaspoonful of salt from the kitchen dresser is a perfectly satisfactory rectal saline, and may be repeated two-hourly, and will do much to replace the lost fluid.

In all cases of haemorrhage, it is important to maintain the body temperature, and warm blankets, hot bottles, and a warm room can always be provided.

When there has been a serious depletion of the total volume of blood, it is important that as good a supply as possible be maintained to the vital centres in the medulla, and if the foot of the bed be raised on chairs, so that the head is lower than the abdomen, the blood that is available has more chance of going to the brain than if the patient is flat.

The available blood in the body can be concentrated in the more important situations by firm bandaging of the limbs, starting at the extremities, and bandaging the whole limb firmly enough to exsanguinate the limb. Subsequent treatment will consist of continuing the ecbolics.

\section{OVARIAN DYSFUNCTION}

\author{
By E. CHALMERS FAHMY, F.R.C.S.(Ed.), F.R.C.P.(Ed.), F.R.C.O.G. \\ (Asst. Gyn. Surg., Royal Infirmary, Edinburgh, etc.)
}

In the last quarter of a century no branch of medicine has received more intensive study than that concerned with the sex hormones; and although considerable knowledge has been acquired, a great deal remains to be clarified. Before discussing the disorders of ovarian function, it is worth while considering briefly the known facts regarding the physiology of the ovary.

\section{Hormones of the Ovary.}

There are two hormones produced'by the ovary, one called oestradiol, the other progesterone." Oestradiol (often termed "Oestrin," or the "Oestrogenic hormone") is produced in both the Graafian follicle of the ovary and in the corpus luteum. Prior to puberty, the follicles enlarge, but not to full maturity; little hormone is produced, but sufficient to aid in the development of the secondary sex characteristics. From puberty to the menopause the monthly maturation of the follicles is accompanied by the regular production of oestradiol. This hormone exerts an important trophic influence on all the genital tissues leading to full development of the organs; it stimulates the pelvic blood circulation, and activates the endometrium and muscle of the uterus. Under this oestrogenic influence the quiescent endometrium thickens, and the glands proliferate during the first half of the menstrual cycle. In the middle of the cycle; the mature Graafian follicle ruptures and allows the ovum to escape; that is to say, ovulation occurs. The follicle is then rapidly transformed into the corpus luteum, which not only continues to produce oestradiol, but also to produce the hormone, progesterone. The latter converts the endometrium of the proliferative phase into the secretory phase, for the corpus luteum hormone alone can stimulate the glands to full secretory activity. The pre-menstrual state of the uterine mucosa is brought about by the combined action of the two ovarian hormones, 
and the mucosa thus becomes ready to receive and nourish a fertilised ovum. If pregnancy does not occur, the corpus luteum atrophies and menstruation occurs. If pregnancy does occur, the corpus luteum continues for some time to enlarge and to secrete quantities of progesterone and oestradiol, both of which are necessary for the continuance of gestation. The rapid growth and tonicity of the uterine musculature is under the influence of oestradiol; while progesterone influences markedly the decidual reaction of the endometrium, the secretion of which nourishes the embedded embryo in its early phase. Progesterone, too, may have the faculty of preventing too great contractile activity of the uterine muscle. As the corpus luteum of pregnancy fades from the second or third month onwards the placenta takes on its function and secretes both oestradiol and progesterone.

\section{Anterior Pituitary Gland.}

The ovary is only one of the chain of endocrine glands which interact on one another; and it is now known that the ovary is governed and controlled by the activity of the anterior pituitary gland. This gland produces "gonadotropic hormones" which directly stimulate the growth of the ovarian follicle, the occurrence of ovulation, and the formation of the corpus luteum; but they do not directly act on the uterus. All the ovarian functions, therefore, are dependent for their regular and balanced activities on the anterior pituitary gland. But the traffic is not all one-way, for the pituitary can be inhibited by the ovarian oestrogenic hormone, the production of which the former stimulates. It has been well stated that the anterior pituitary gland is the motor that drives the ovary.

Of the other endocrine glands the thyroid and the adrenal appear to have most influence over ovarian activity, though indirectly.

\section{OVARIAN DYSFUNCTION}

Dysfunction will occur when there is significant alteration in the relative quantity of hormone produced as between oestradiol and progesterone. Actual excess of hormone is $<$ uncommon; the clinical disturbance is more commonly the result of unduly prolonged influence 8 of one hormone when the other is deficient or absent. Oestrogenic hormone may be actinge when progesterone is completely in abeyance; but the reverse is impossible, for corpus luteumo formation occurs only after ovulation. With few exceptions, ovarian dysfunction is secondary? to imperfect action of the anterior pituitary gland; but primary ovarian failure (inability of the ovary to respond to pituitary stimulation) may occur, though rarely. The clinical manifestations of dysfunction may be associated with ovaries of normal size, with those slightly enlarged and cystic, or with those grossly enlarged by tumour formation. An accurate diagnosis of ovarian behaviour can seldom be made without histological examination of endometrial tissue removed by curettage; and in assessing the significance of the endometrial picture, it is necessary to make due allowance for time relationship between the onset of symptoms and the date of the investigation. These facts must be known to the pathologist to enable a correct report to be submitted. In many instances, it is necessary to determine the amount of ovarian and pituitary hormones present in the blood and urine of the individual, and these investigations may have to be repeated more than once. These bio-chemical assays are by no means easy to undertake, and cannot, therefore, be carried out as a routine. Progesterone cannot yet be determined in the blood; its presence is gauged by the amount excreted in the urine of its derivative pregnanediol. If pregnanediol is present, activity of corpus luteum may be presumed.

The clinician must, therefore, realise that whereas some cases of dysfunction may be assessed fairly accurately by simple measures, others require extended and specialised diagnostic procedures. Both hormones must, of course, be considered in any case of dysfunction, but reference will here be made to each separately according as to which is the predominating factor.

\section{Undue Influence of Oestrogenic Hormone.}

This is common and often manifested by prolonged and irregular uterine bleeding. In well-marked cases ovulation fails to occur and corpora lutea, therefore, do not develop. As a result, oestradiol is unopposed and leads to excessive proliferation of the endometrium and congestion of the uterine musculature. "The endometrium may become polypoidal and the uterine wall hypertrophied. The older clinicians termed this "polypoidal endometritis," but we now 
know that it is a true endocrine disorder. Often, but not always, one or other ovary contains large, cystic Graaifian follicles which are rich in oestradiol. "Metropathia Haemorrhagica" is the term now employed to describe this condition. After one or two missed periods, bleeding occurs and may carry on for weeks in varying degree - the symptoms sometimes leading to a diagnosis of incomplete abortion until curettage settles the point. Haemorrhage of this type is unassociated with pain-a point of importance. The blood remains charged with oestradiol until it exerts an inhibitory effect on the pituitary, which effect, in turn, lessens the production of oestradiol. It is at this stage, of oestrogenic fall, that haemorrhage commences. The condition is most common in women over thirty-five years of age, but it also occurs at puberty, when the young child may bleed intermittently for weeks - the so-called "menorrhagia of puberty."

Reference may be made here to the special type of ovarian tumour, granulosa-celled tumour, that produces large quantities of oestrogenic hormone leading to endometrial hyperplasia and uterine haemorrhage; no progesterone is produced. The tumour may develop in young girls prior to puberty, and in women at or after the menopause; it is often benign, and its removal leads to cessation of bleeding.

Undue influence of oestrogenic hormone may; however, be manifested even when corpus luteum formation occurs, but when progesterone production is insufficient. Menorrhagia is, perhaps, more common than metrorrhagia, though menstruation may be irregular. Relative excess of oestrin over progesterone may be responsible for habitual menorrhagia, even though the uterus shows no clinical enlargement. The problem is an ovarian one, not uterine. Incidentally, the view has recently been expressed that uterine fibroids and pelvic endometriosis may develop as the result of relative persistent oestradiol excess over a long period.

Alteration in the menstrual rhythm from monthly to three-weekly type occurs in many individuals. It is common after childbirth even when recovery has been smooth and complete; but it also occurs after illness, or even without assignable cause. It results from a change in the ovarian activity as regards time of ovulation, which occurs earlier in the cycle than formerly. Both hormones are adequately produced, but the cycle is speeded up. The shortened cycle may persist, but often there is return spontaneously to the original type. This is an example of altered function rather than one of dysfunction.

\section{Lack of Oestrogenic Hormone, or Diminished Production.}

Scanty hormone will result in amenorrhoea or scanty menstruation. It may appreciably delay the onset of menstruation at puberty even though the secondary sexual characters are well developed. One who menstruates late (at I9 or 20 years) may have a hypoplastic uterus with a small conical cervix; scanty menstrual loss may persist, or full development of the uterus may later occur. Women with hypoplastic uteri tend to be sterile and may, or may not, have primary dysmenorrhoea.

Rarely, the ovary at puberty fails to respond to adequate pituitary stimulation, i.e. primary ovarian failure. Primary amenorrhoea will persist until response occurs. In other cases, after years of normal menstruation, secondary amenorrhoea may occur without apparent cause, due to poor ovarian function with diminished hormone production. If investigations always revealed identical findings, then the true explanation of the amenorrhoea would be readily determined. But though the ovary must be failing in its function, even though temporarily, curettage of the uterus reveals no uniformity of appearance in the endometrium. One would expect to obtain a thin, inactive mucous membrane-but this is not always the case. Secondary amenorrhoea may be associated with a normal resting endometrium, indicating that oestradiol is being produced in sufficient quantity in the ovary to exert at least some trophic influence on the endometrium, and so prevent its atrophy.

Some women over considerable periods of time exhibit a scanty loss of blood for a day or two midway between the periods-i.e. at the time of ovulation, and concern is usually expressed at its occurrence. The cause is uncertain, but the condition may be associated with diminished oestrogenic hormone. The writer has carried out curettage at the onset of the bleeding and found an endometrium of subnormal proliferative activity; and occasionally the administration of oestrin prevents this so-called "ovulation haemorrhage." The matter is still, however, unsettled. Whether the bleeding precedes, coincides with, or follows ovulation is undetermined. 
It is common knowledge that severe illness or under-nourishment may alter the menstrual rhythm or lead to amenorrhoea; but cessation of the menses also occurs in women in perfect health. The ovarian dysfunction reflects an altered pituitary function; but why the latter occurs is usually difficult to determine. Frohlich's disease, Cushing's syndrome, and Simmond's disease are examples of clinical states with general symptons of pituitary origin, and these can $\vec{\Rightarrow}$ be diagnosed by extended investigation; but the onset of secondary amenorrhoea or scanty $\stackrel{0}{9}$ menstruation is more often not associated with definite pituitary srmptoms. Thyroid dvsfunc: tion certainly affects the ovary, probably via the pituitary, just as does an adrenal tumour: biochemical investigations will help here in the elucidation of the problem.

One special ovarian tumour, arrhenoblastoma, produces the male-type of hormone leading to amenorrhoea and masculine attributes. It forms another instance of ovarian dysfunction \& due to a neoplasm, removal of which relieves the symptoms.

Oestradiol production fades at the menopause when the ovaries gradually cease to function. One result is a tendency to vaso-motor disturbances, such as flushings, due to the anterior pituitary hormones having little or no oestradiol to inhibit their too great activity. It is for this reason that oestrogenic hormone is administered to control hot flushes, and with much success as a rule. Post-menopausal vaginitis and vulval skin lesions, too, have, as a factor in their origin, lack of oestrogenic hormone. Oestrin therapy is valuable, also, in such cases.

\section{Undue Influence of Progesterone.}

This hormone is seldom present in such excessive quantity as to produce symptoms. It is 은 well to remember that the corpus luteum produces not only progesterone but also oestritliol, so that excess of the former is unlikely. It is, however, held by some that hyperactivity of the $\infty$ corpus luteum may cause an excessively thick premenstrual endometrium, so that the tissue $\frac{\mathbb{D}}{0}$ is cast off from the uterus at menstruation with difficulty and in pieces of some size, instead of in small crumblings - the condition of membraneous dysmenorrhoea.

There is, however, one definite, though infrequent, abnormality of the corpus luteum clinical importance-the corpus luteum cyst. Dysfunction is manifested in a train of symptons that may closely simulate those of ectopic pregnancy, even including a weak positive Asch in Zondek test for pregnancy. This A.Z. test depends on the amount of anterior pituitary-lik? hormones in the urine; it does not depend on the excretion of pregnanediol, the derivative of progesterone.

\section{Lack of Progesterone or Diminished Production.}

When ovulation fails to occur, corpora lutea cannot develop, and as a result the endometrium will not develop the secretory activity which characterises the later phase of the premenstrual endometrium. Sterility, therefore, is the lot of the individual. Curettage, performed just before menstruation is due, will remove tissue which will reveal whether secretory changes are present or not. If not, then ovulation is not occurring, even though menstruation - "anovular menstruation"-is regular and clinically normal. The unopposed oestradiol from the follicles is not, in such a case, leading to irregular uterine bleeding as in the clearly defined condition, metropathia haemorrhagica. The patient is clinically normal, sterility being the one complaint. To stimulate ovulation, anterior pituitary-like hormone from the blood serum of pregnant mares is employed. Anterior pituitary hormone commercial preparations mostly. 윽 contain the factor which stimulates follicle development; few, at present known, contain the $D$ factor that directly stimulates ovulation. Pregnant mares' serum does, in some cases, have ․ㅡ․ clinical value here.

Progesterone in insufficient quantity will lead to difficult $y$ in embedding in the endometrium of a fertilised ovum, for the endometrium is not fully prepared for its reception. But if implantation does occur, there is a tendency for early abortion. Lack of sufficient ovarian progesterone is accepted as one cause underlying habitual abortion. About the third month of pregnancy the placenta takes on the function of supplying both oestradiol and progesterone to safeguard the pregnant uterus, after the normal retrogression of the corpus luteum has begun. It is $\stackrel{D}{\mathscr{D}}$ in cases of habitual abortion and threatened abortion that progesterone is to-day emplored more than in any other condition; jt not only aids the full development of the decidua of 
pregnancy, but it may, possibly, inhibit to some extent activity of the uterine muscle. Its clinical value is greatest in the first few weeks of pregnancy in virtue of its effect on the decidual tissue; there is little evidence of its value later on in the human being.

It is impossible to discuss within the limits of this article the full therapeutic use of ovarian hormones or the treatment of dysfunction in general, though mention has been made of one or two conditions in which they may be employed successfully. Many cases of dysfunction respond to general medical and dietetic measures, and some respond to thyroid administration. The pituitary and ovarian hormones should be employed only after most careful consideration and, if necessary, full detailed investigation. Commercial preparations of synthetic oestrogens are now so inexpensive that these have perhaps been employed without full appreciation of their probable value. Haphazard employment is to be condemned, for harm, rather than good, may result. The first line of attack on menstrual disorders is a general examination of the patient; such examination may demonstrate the need for measures other than hormone preparations.

\title{
THE DIFFICULTIES AND DANGERS OF THE PRIMIPAROUS WOMAN
}

\author{
By MORRIS DATNOW, F.R.C.O.G. \\ (Hon. Asst. Surgeon, Liverpool Maternity Hospital, and Women's Hospital, Liverpool) \\ Introduction
}

Most obstetrical problems are common to all women, multiparae and primigravidae alike, there are, however, features which can be regarded as special to potential mothers carrying their first child, and it is with these clinical conditions that this paper is essentially concerned.

The care of the primigravida is yet the obstetrician's most urgent and anxious problem, for her safe delivery prevents many undesirable sequelae, leading to sterility, disability, injuries, invalidism, and a fear of bearing more children. This psychological state is difficult to rectify and naturally harmful to the national economy. In practice many women are encountered who only have or had one child, and state that they had such a bad time in the confinement that they really could not face another, although they would very much have liked a family.

There has been considerable progress in antenatal care and diagnosis, especially with refer-. ence to X-ray examinations, biological tests, also advances in obstetrical technique, improved anaesthesia, operative technique, greater institutional facilities with more prolonged teaching and training of both midwives and students, and post-graduate courses. New drugs and also improved old ones. All these factors must play their part in lessening morbidity and mortality. Tocergometry, hysterography and the counting of labour pains are more recent investigations which have been interrupted by prevailing conditions, and the future will possibly demonstrate their value as the results observed so far already indicate.

Notwithstanding the amount of the clinical research that has been carried out there are as yet many unknowns to cause anxiety during pregnancy and labour for the primigravida. Hospital facilities for the lying-in woman are far from sufficient, and there still is a tendency to foster domiciliary midwifery in districts which only have antenatal clinics supervised by general practitioners without adequate qualification for this very specialised branch of medical practice. In some localities primigravidity is not looked upon as sufficient indication for institutional delivery. This is greatly to be deprecated, especially with the imperfect home help that is available at present. 